\title{
Colorectal motor and sensory function after hysterectomy
}

\author{
Eduard A. van Hoboken • Frederik G. M. Timmermans • \\ Patrick P. J. van der Veek • Philomeen T. Weyenborg • \\ Ad A. M. Masclee
}

Accepted: 31 December 2013 / Published online: 14 January 2014

(C) Springer-Verlag Berlin Heidelberg 2014

\begin{abstract}
Purpose Women may develop constipation after hysterectomy. The pathophysiology and underlying mechanisms are poorly understood. They may originate from either neural damage of rectum and colon or changes in anatomical constellation of the remaining pelvic organs. The aim of this study is to evaluate sensory and motor functions of rectum and colon in women with newly developed constipation after hysterectomy in comparison with women without constipation and healthy controls after hysterectomy .

Methods Barostat measurements were performed in posthysterectomy women with constipation (PH-C), without constipation (PH-NC), and healthy controls $(n=10$, every group). Outcome measures were rectal and colonic compliance (millilitre per millimetre of mercury), rectocolonic perception in reaction to mechanical distension (millimetre; VAS scores) and rectocolonic reflex (millilitre per millimetre of mercury).

Results No differences in rectal or colonic compliance were observed. Urge perception due to rectal distension increased significantly in controls (from $7 \pm 5$ to $41 \pm 10 \mathrm{~mm} ; p<0.05$ ) and PH-NC group (from $3 \pm 1$ to $24 \pm 9 \mathrm{~mm} ; p<0.05$ ), but not
\end{abstract}

\section{E. A. van Hoboken · F. G. M. Timmermans - A. A. M. Masclee Department of Gastroenterology, Maastricht University Medical Center, Maastricht, The Netherlands}

E. A. van Hoboken • F. G. M. Timmermans • P. P. J. van der Veek Department of Gastroenterology-Hepatology, Leiden University Medical Center, Leiden, The Netherlands

\section{E. A. van Hoboken $(\bowtie)$}

Department of Gastroenterology, Rode Kruis Ziekenhuis, Vondellaan

13, 1942 LE Beverwijk, The Netherlands

e-mail: evanhoboken@rkz.nl

\section{P. T. Weyenborg}

Department of Gynaecology, Leiden University Medical Center, Leiden, The Netherlands in PH-C patients ( $1 \pm 1$ to $11 \pm 5 \mathrm{~mm}$; ns). In healthy controls and the PN-NC group, respectively, 100 and $70 \%$ of subjects reached the minimal threshold value for urge of $10 \mathrm{~mm}$ during the isobaric distension sequence. In the PH-C group, only two subjects $(20 \%)$ reached this threshold $(p<0.05)$. Rectal pain perception, phasic colonic motility and the rectocolonic reflex were intact in all three groups.

Conclusions Colorectal motor and sensory function is generally well preserved in women with constipation after hysterectomy. It is unlikely that the symptom of constipation after hysterectomy has been caused by iatrogenic neuronal damage in these patients.

Keywords Hysterectomy · Constipation .

Visceroperception $\cdot$ Rectocolonic reflex

\section{Introduction}

Hysterectomy is a commonly performed surgical procedure. The decision to perform hysterectomy for non-malignant indications is based on the anticipated benefit with respect to symptoms and quality of life while minimising risks and complications. Potential disadvantages of hysterectomy include not only changes in urinary and sexual functions but also in bowel function. Although recent prospective studies have shown that the overall prevalence of constipation does not increase after hysterectomy, subgroups of women have been identified with newly developed constipation after hysterectomy [1-3].

With respect to this newly developed constipation after hysterectomy, two potential pathophysiological mechanisms have been proposed as follows: (1) neural damage related to the surgical intervention and (2) mechanical changes in the spatial relationship of the organs in the pelvic region due to removal of the uterus. 
Results of studies evaluating rectocolonic motor and sensory function in posthysterectomy patients are not uniform. Prior et al. [4] investigated anorectal function in 26 women before and after hysterectomy, using anorectal manometry and rectal barostat. Rectal sensitivity tended to be increased after hysterectomy, but static compliance was not influenced by hysterectomy. In a study by Goffeng et al. [5], no significant changes in anal sphincter pressures and whole-gut transit time after hysterectomy could be demonstrated. Both studies have been performed in women after hysterectomy without taking into account the presence of constipation. Varma [6], who studied women with constipation after hysterectomy, observed a significantly increased rectal volume and compliance together with disturbances in rectal sensory function.

In a previous study, we have shown that visceroperception is reduced in patients with slow transit constipation: while in IBS patients with the constipation subtype, the perception to rectal distension is increased compared to healthy controls [7].

The rectocolonic reflex, that is reflex inhibition of more proximal colonic motility during rectal distension has previously been described and demonstrated in humans. This reflex requires intact rectocolonic sensory and motor function. Measurement of this reflex was therefore included in the present study protocol. The aim of our study was to evaluate the sensory and motor function of the rectum and colon in (1) women after hysterectomy with newly developed constipation (2) women after hysterectomy with persistent normal bowel habit (no constipation) and (3) healthy female controls. Our hypothesis was that patients with newly developed constipation posthysterectomy have a disturbed rectocolonic motor and/or sensory function.

\section{Materials and methods}

\section{Participants}

A database comprising all patients who had undergone a hysterectomy at the Gynaecology Department of Leiden University Medical Center between 2 and 4 years prior to the study was used to select patients for inclusion. All cases of hysterectomy performed because of malignancy, premalignancy, or bleeding after childbirth were excluded. The prevalence of constipation before and after hysterectomy in the remaining group was evaluated retrospectively according to the Rome-III criteria [8]. From the group of women with newly developed constipation after hysterectomy and the group of women that had never experienced constipation neither before nor after hysterectomy, ten participants were asked to participate and were included. Furthermore an agematched control group $(n=10)$ of women that did not undergo hysterectomy and did not experience constipation, was recruited by advertising in a local newspaper. None of the participants had undergone abdominal surgery apart from hysterectomy. None of the participants exhibited comorbidity that could possibly interfere with colorectal function. Participants were asked to stop medication that could possibly influence colorectal function at least 1 week prior to the measurements.

The study protocol had been approved by the ethical committee of the Leiden University Medical Center and written informed consent was obtained from all patients.

\section{Estimated sample size}

This study was designed as a proof-of-concept study for the hypothesis that constipation after hysterectomy would have its origin in surgical injury of the enteric nerve system. As a primary outcome parameter for confirmation of intact enteric neuronal control, the presence or absence of the rectocolonic reflex was used. In a former study by our group, we have shown that in healthy volunteers, a significant increase in colonic balloon volume is present upon rectal balloon distension. Using these data as a reference, a statistical power of sample size of $n=10$ in each group was sufficient to have a $90 \%$ chance of demonstrating a rectocolonic response at the $5 \%$ level of significance using a $t$-test.

\section{Barostat}

We used a double barostat assembly, as we have described before [9]. Two electronic barostats (Visceral Stimulator, Medtronic, Skovlunde, Denmark) were used to measure volume changes in the rectum and colon and to perform isobaric distensions. Pressure and volume were measured using specialised software (Polygram for Windows SVS module, Medtronic, Skovlunde, Denmark).

Experimental design

Bowel preparation consisted of 2-L polyethylene glycol (Klean-Prep ${ }^{\circledR}$, Norgine BV, Amsterdam, The Netherlands) on the evening before the experiment. After an overnight fast, patients received a tap water enema. Colonoscopy into the descending colon was performed and a flexible guidewire was introduced endoscopically up to the splenic flexure. After withdrawal of the colonoscope, a barostat catheter was introduced into the descending colon under fluoroscopic control, after which, the guidewire was removed and the second barostat catheter was introduced into the rectum. The participant was then placed in a supine position in a hospital bed.

Barostat procedure

Both barostat bags were used (1) to distend the rectum or descending colon and (2) to measure tonic and phasic volume 
changes. Total time of the barostat procedure was $160 \mathrm{~min}$. The procedure consisted of the following components.

\section{Minimal distending pressure}

First, minimal distending pressure (MDP) was determined in colon and rectum during a ramp distension procedure, in which intrabag pressure was elevated from 5 to $30 \mathrm{mmHg}$, with steps of $1 \mathrm{mmHg}$ per minute. MDP is defined as the minimal pressure that provides an ongoing intravisceral bag volume of more than $30 \mathrm{~mL}$.

\section{Adaptation}

Both bags were set to maintain a pressure of MDP $+3 \mathrm{mmHg}$ (operating pressure) for $15 \mathrm{~min}$ to allow adaptation.

\section{Ramp distension}

Rectal bag pressure was gradually increased from 5 to $40 \mathrm{mmHg}$ with steps of $1 \mathrm{mmHg}$ per minute. Then an identical procedure was repeated in the sigmoid bag. During both rectal and colonic distension protocols, perception of urge and pain were scored at regular intervals by means of visual analogue scales (VAS) from 0 to $100 \mathrm{~mm}$.

\section{Rectocolonic reflex}

Six consecutive isobaric distensions were given in the rectum at pressures of $10,15,20,25,30$, and $35 \mathrm{mmHg}$, respectively, each for $5 \mathrm{~min}$. Each distension was followed by a 5 -min long break with intrabag pressure at $5 \mathrm{mmHg}$. During distensions, both pressure and volume were measured in the barostat bag, located in the sigmoid.

Data analysis

Barostat measurements were performed and analysed using Polygram for Windows 2.04. Compliance was defined as the maximum increase in volume reached with $1-\mathrm{mmHg}$ pressure rise during the ramp distension (i.e., the maximum slope of the pressure-volume curve) [10]. Bag volume during isobaric distensions was defined as the mean bag volume of the last 2 min before the next distension step. Phasic volume events (PVEs) were defined as a decrease in volume of at least $10 \%$ relative to baseline with duration of 10-60 s. PVEs are an expression of peristaltic contractions in the colonic wall adjacent to the barostat bag [11]. VAS scores were quantified in millimetres (ranging from 0 to $100 \mathrm{~mm}$ ). Maxima and thresholds for urge and pain were compared between different subgroups. In order to eliminate the possible effect of differences in compliance on visceroperception, correlation analysis was performed between VAS scores for perception and wall tension, calculated using LaPlace's law for cylindrical vessels.

Data are presented as mean \pm SEM in case of numerical variables and as percentage in case of categorical variables. Statistical comparison between the three groups was performed using ANOVA tests with post hoc Tukey test in case of numerical variables and with chi-square tests in case of categorical variables. $P<0.05$ was considered as statistically significant.

\section{Results}

Thirty women agreed to participate in the study: group A, ten women with newly developed constipation posthysterectomy ( $\mathrm{PH}-\mathrm{C})$, group $\mathrm{B}$, ten non-constipated women posthysterectomy (PH-NC) and group $\mathrm{C}$, ten control subjects. Demographic characteristics of all participating women are shown in Table 1. No differences in demographics between the three groups were present, apart from a significantly higher BMI in the posthysterectomy groups: $26.9 \pm 1.8$ (PH-C) and $27.1 \pm 0.9$ (PH-NC) vs $22.8 \pm 0.9(\mathrm{C}) \mathrm{kg} / \mathrm{m} 2(p<0.05)$. As shown in Table 1, constipation in PH-C patients is characterised predominantly by symptoms that resemble outlet obstruction (i.e., straining, incomplete evacuation, a feeling of anorectal obstruction, and the need for digital evacuation).

Motor function

Colon

Compliance in the colon was $22 \pm 7 \mathrm{~mL} / \mathrm{mmHg}$ in $\mathrm{PH}-\mathrm{C}$ patients, $15 \pm 2 \mathrm{~mL} / \mathrm{mmHg}$ in PH-NC group, and $16 \pm 3 \mathrm{~mL} /$

Table 1 Demographics

\begin{tabular}{llll}
\hline Group & PH-C & PH-NC & HV \\
\hline Age & $45 \pm 2$ & $51 \pm 3$ & $49 \pm 2$ \\
BMI (kg/m) & $27 \pm 2$ & $27 \pm 2$ & $23 \pm 1 *$ \\
Indication for hysterectomy & $9 / 1$ & $8 / 2$ & \\
$\quad$ (bleeding/prolapse) & & & \\
Surgical approach (abdominal/vaginal) & $8 / 2$ & $6 / 4$ & \\
Time after surgery (years) & $3 \pm 1$ & $4 \pm 1$ & \\
Defecation frequency <3/week (\%) & $33 \%$ & $0 \%$ & $0 \%$ \\
Straining (\%) & $100 \%$ & $20 \%$ & $0 \%$ \\
Incomplete evacuation (\%) & $88 \%$ & $11 \%$ & $0 \%$ \\
Anorectal obstruction & $56 \%$ & $0 \%$ & $0 \%$ \\
Digital evacuation & $44 \%$ & $0 \%$ & $0 \%$ \\
\hline
\end{tabular}

$P H-C$ constipated posthysterectomy; $P H-N C$ non-constipated posthysterectomy; $C$ healthy controls

${ }^{*} p<0.05$ HV vs. Both PH-C and PH-NC 
$\mathrm{mmHg}$ in controls. Compliances did not significantly differ between the three groups.

The frequency of PVEs per hour was $37 \pm 7$ in PH-C patients, $30 \pm 6$ in the $\mathrm{PH}-\mathrm{NC}$ group, and $29 \pm 5$ in the controls. No significant differences between the three groups were observed.

\section{Rectum}

Compliance of the rectal wall was $21 \pm 5 \mathrm{~mL} / \mathrm{mmHg}$ in $\mathrm{PH}-\mathrm{C}$ patients, $13 \pm 1 \mathrm{~mL} / \mathrm{mmHg}$ in the PH-NC group and $19 \pm 2 \mathrm{~mL} /$ $\mathrm{mmHg}$ in the controls. Compliances did not significantly differ between the three groups (Fig. 1).

Sensory function

\section{Colon}

During the ramp distension procedure in the colon, perception scores for urge showed small increments without any relevant differences between groups. In controls, urge increased from $1 \pm$ 1 at $p=5 \mathrm{mmHg}$ to $12 \pm 6$ at $p=30 \mathrm{mmHg}$; (ns) in the PH-NC group from $1 \pm 0$ to $8 \pm 4$; and in the PH-C group from $3 \pm 2$ to $3 \pm$ 2 (ns). No changes in pain perception were observed.

\section{Rectum}

During isobaric distensions in the rectum, urge perception scores in the pressure range 5 to $30 \mathrm{mmHg}$ increased significantly in controls (from $7 \pm 5$ to $41 \pm 10 \mathrm{~mm} ; p<0.05$ ) and PH$\mathrm{NC}$ group (from $3 \pm 1$ to $24 \pm 9 \mathrm{~mm} ; p<0.05$ ), but not in PH-C patients ( $1 \pm 1$ to $11 \pm 5 \mathrm{~mm}$; ns). In healthy controls and the PN-NC group, respectively. 100 and $70 \%$ of subjects reached the minimal threshold value for urge of $10 \mathrm{~mm}$ during the isobaric distension sequence. In the PH-C group, only two

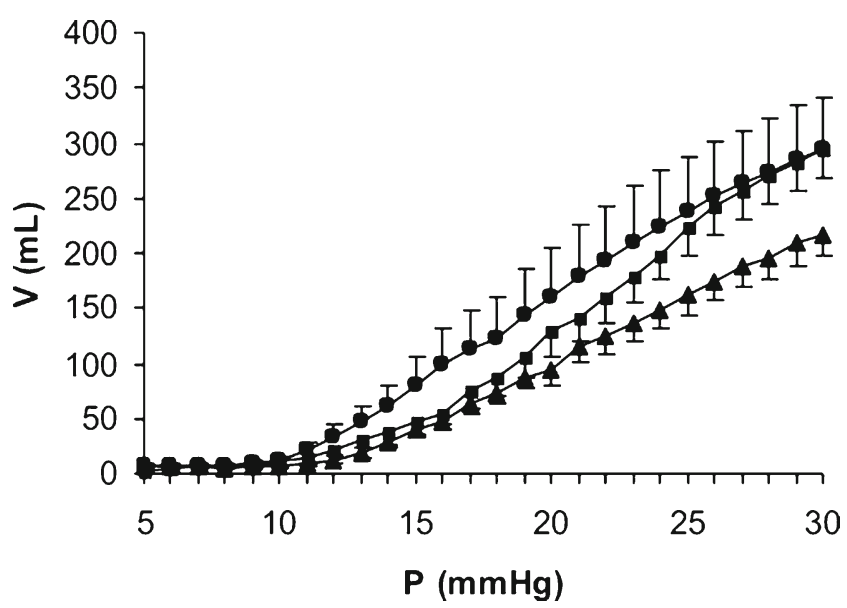

Fig. 1 Rectal compliance. Rectal volume is plotted against rectal pressure. Note that PH-C (dots) do not significantly differ from PH-NC (triangles) and healthy controls (squares) subjects $(20 \%)$ reached this threshold $(p<0.05)$. Figure 2 shows that the correlation between urge (millimetre) and wall tension (gram per centimetre) is equal in healthy controls and PH-NC, but is significantly lower in PH-C patients.

No significant increase in pain perception was observed during ramp distension in any of the three groups (data not shown).

\section{Surgical approach}

A post hoc analysis was performed, comparing patients that underwent an abdominal hysterectomy $(\mathrm{AH})$ to those that underwent a vaginal hysterectomy $(\mathrm{VH})$, concerning compliance and urge perception. Compliance was $15.5 \pm 1.0 \mathrm{ml} /$ $\mathrm{mmHg}$ in $\mathrm{VH}$ vs $17.6 \pm 4.5 \mathrm{~mL} / \mathrm{mmHg}$ in $\mathrm{AH}(\mathrm{ns})$.

The percentage of subjects that did not reach the urge threshold of $10 \mathrm{~mm}$ was $50 \%$ in the $\mathrm{VH}$ group and $58 \%$ in the $\mathrm{AH}$ group (n.s.).

Rectocolonic reflex

During progressive intermittent phasic pressure distensions (ranging from 15 to $35 \mathrm{mmHg}$ ) in the rectum, colonic volumes progressively increased (Fig. 3). This phenomenon, called the rectocolonic reflex, was present in all three groups. In the PH$\mathrm{C}$ group as well as in healthy controls, the reflex increase in volume first occurred at the distension step of $20 \mathrm{mmHg}$, whereas in PH-NC group, the reflex first occurred at higher

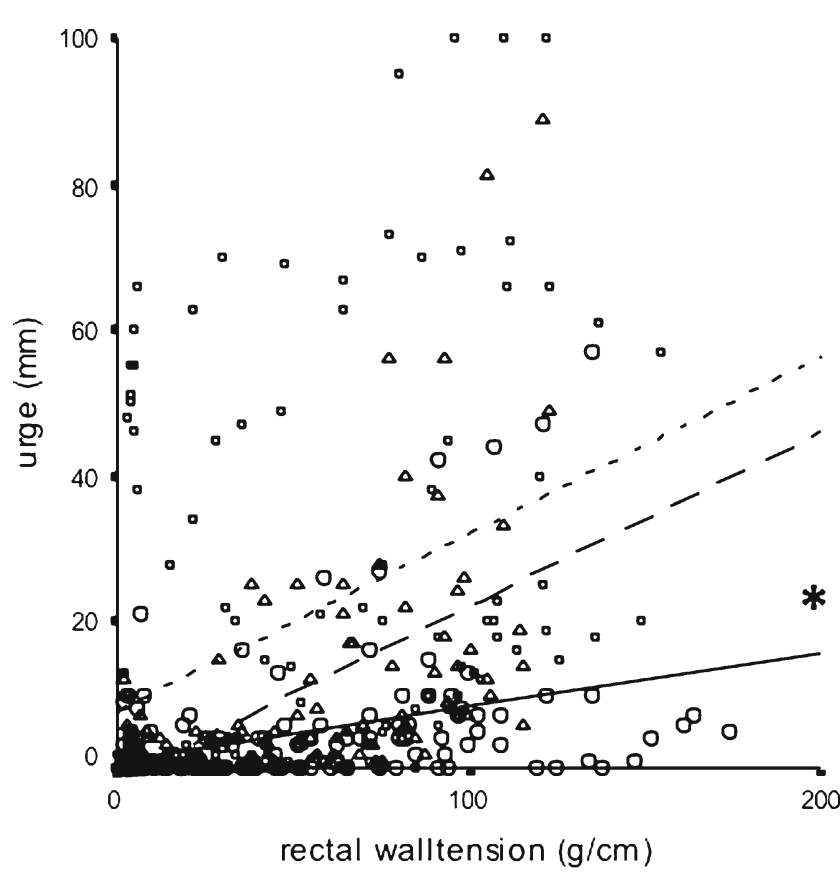

Fig. 2 Urge perception. Urge perception is plotted against rectal wall tension during isobaric (rectal) distensions. In all three groups, the perception of urge increases with increasing wall tension. However, this increase is smaller in PH-C (dots/solid line) than in PH-NC (triangles, rough dotted line) and HV (squares; dotted line). ${ }^{*} P<0.05 \mathrm{PH}-\mathrm{C}$ vs both other groups 


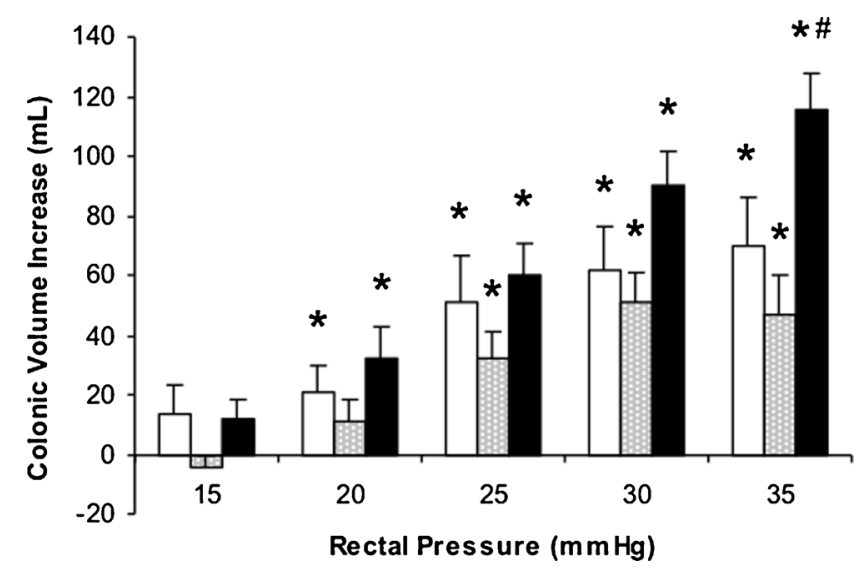

Fig. 3 Rectocolonic reflex. During phasic distensions of the rectum an increase in colonic bag volume was observed in PH-C (black), PH-NC (grey) and controls (white). This phenomenon is called the rectocolonic reflex. In $\mathrm{PH}-\mathrm{C}$, the volume increase was significantly higher than in $\mathrm{PH}-$ $\mathrm{NC}$ during the highest distension $(35 \mathrm{mmHg}) .{ }^{*} p<0.05$ vs basal; $\# p<0.05$ $\mathrm{PH}-\mathrm{C}$ vs PH-NC

pressure, which is at the $25-\mathrm{mmHg}$ distension step. The magnitude of the volume increase tended to be higher although not significantly in the PH-C group in comparison with the other two groups. The difference was statistically significant only between PH-C and PH-NC $(115 \pm 12 \mathrm{~mL}$ vs $47 \pm$ $14 \mathrm{~mL}$ at $P=35 \mathrm{mmHg} ; p>0.05$ ).

\section{Discussion}

In this explorative study, we have evaluated colorectal motor and sensory functions in constipated posthysterectomy patients, in comparison with non-constipated posthysterectomy patients and healthy controls. It was found that in both groups, after hysterectomy, colorectal motor function (tonic as well as phasic motility) is preserved. The ability of the colon to relax upon rectal mechanical stimulation, the rectocolonic reflex, was intact. In constipated women, this response was even more pronounced, compared to the two other groups. Considering sensory function, perception of urge in response to mechanical distension of the rectum was diminished in women with posthysterectomy constipation in contrast to nonconstipated women and control subjects.

The aim of our study was primarily to test whether the rectocolonic response remains intact in women with constipation after hysterectomy. The sample size calculation of the study was based on this item. However, it cannot be excluded that for the other parameters we evaluated in this study, a typeII error causes that true differences are not detected.

While data from retrospective studies have suggested that constipation frequently occurs after hysterectomy $[4,5]$, recent prospective studies indicate that overall, the prevalence of constipation after hysterectomy is not different from the prehysterectomy condition. While this holds true at group level, subgroups can be identified in which constipation is resolved after hysterectomy and patients with newly developed constipation after the procedure. The cause of this de novo constipation is unknown. Two potential mechanisms have been proposed as follows: (1) neural damage and (2) mechanical changes in the three-dimensional orientation of the organs in the pelvic region due to the hysterectomy.

The innervation of the distal part of the colon and the rectum is provided by two principally extra-intestinal nerve systems: (1) mesenteric nerves, originating from the prevertebral ganglia and (2) pelvic nerves, originating from the sacral segments of the spinal cord. Both nerve conduits provide motor as well as sensory innervation to and from the gut. Although hysterectomy for benign indications always is performed according to a nerve-sparing method, aiming to spare the innervation of the rectum, it is still possible that nerves have been damaged accidentally.

The only difference we observed between constipated and non-constipated women after hysterectomy was a reduced perception of urge. This rectal hyposensitivity for urge may result from nerve damage, but rectal motor function was preserved. The observed hyposensitivity in women with constipation after hysterectomy resembles that in patients with slow transit constipation. Similar to the situation in slow transit constipation, the relationship between hyposensitivity and the development of constipation posthysterectomy is not clear. Both rectal hyposensitivity as well as the more pronounced response of the colon in the rectocolonic reflex may be consequence rather than cause of constipation. However, the preservation of the rectocolonic reflex after hysterectomy clearly shows that neural innervation of the distal colon and rectum remained intact and therefore it seems unlikely that constipation after hysterectomy results from neural damage.

The question whether the alternative hypothesis that constipation is the result of rearrangement of pelvic organs, is true cannot be fully answered based on our data. However, analysing the complaints of the patients in the $\mathrm{PH}-\mathrm{C}$ group in more detail, it becomes clear that local anorectal factors appear to be involved. As shown in Table 1, the symptoms of the $\mathrm{PH}-\mathrm{C}$ patients in our study point to defecatory dysfunction (outlet obstruction), rather than true (colonic) slow transit constipation. The weight of the removed uterus did not significantly differ between the PH-C and the PH-NC group ( $189 \pm 58$ vs $283 \pm 124 \mathrm{~g}$; ns). No differences were observed between the abdominal vs vaginal route of hysterectomy. Therefore, it is not very likely that the amount of removed tissue, pelvic rest volume, route of surgery etc., have contributed to 'de novo' development of constipation after hysterectomy. These arguments are not in favour of an 'anatomical' explanation of constipation after hysterectomy. Further studies are necessary focussing at the different types of mechanoreceptors in the gut. 
It should be mentioned that the three groups of women in our study differed with respect to their BMI. In the group of healthy controls, the BMI was significantly lower compared to the posthysterectomy patients. Theoretically, BMI may be a confounder, since obesity has been shown to be associated with decreased colonic compliance and decreased pain perception [12]. However, no significant differences in compliance and pain perception between the three groups were observed. Therefore, it is less likely that differences in BMI may have influenced our results. Concerning other baseline characteristics, there were differences, although nonsignificant, between the percentage of abdominal (as opposed to vaginal) procedures between the $\mathrm{PH}-\mathrm{C}$ group and the $\mathrm{PH}-$ $\mathrm{NC}$ group. Furthermore, there was a slight but non-significant difference in the interval between surgery and inclusion in this study between the two groups. It is unlikely that either type of surgery or timing after surgery may have additionally influenced motor and sensory function. Furthermore, to our knowledge, no studies have been published previously, pointing to differences in colorectal motor and sensory function related to type of surgery or timing of surgery. A post hoc analysis of our data did not reveal any effect of both parameters on motor and sensory outcome parameters. Therefore, it is unlikely, that these slight and non-significant differences in baseline characteristics have influenced our results.

In conclusion, we have demonstrated that colorectal motor and sensory function in women with newly developed constipation after hysterectomy is generally well preserved apart from a reduction in rectal urge perception. Colorectal motor and sensory function in these patients does not appear to be procedure related.

\section{References}

1. Roovers JP, van der Bom JG, van der Vaart CH, HysVA Study Group (2008) Hysterectomy does not cause constipation. Dis Colon Rectum 51:1068-72

2. Sperber AD, Morris CB, Greemberg L et al (2009) Constipation does not develop following elective hysterectomy: a prospective, controlled study. Neurogastroenterol Motil 21:18-22

3. Forsgren C, Zetterström J, Lopez A, Nordenstam J, Anzen B, Altman D (2007) Effects of hysterectomy on bowel function: a three-year, prospective cohort study. Dis Colon Rectum 50:1139-45

4. Prior A, Stanley K, Smith AR, Read NW (1992) Effect of hysterectomy on anorectal and urethrovesical physiology. Gut 33:264-267

5. Goffeng AR, Andersch B, Antov S, Berndtsson I, Oresland T, Hulten L (1997) Does simple hysterectomy alter bowel function? Ann Chir Gynaecol 86:298-303

6. Varma JS (1992) Autonomic influences on colorectal motility and pelvic surgery. World J Surg 16:811-819

7. Penning C, Steens J, van der Schaar PJ et al (2001) Motor and sensory function of the rectum in different subtypes of constipation. Scand J Gastroenterol 36:32-8

8. Longstreth GF, Thompson WG, Chey WD, Houghton LA, Mearin F, Spiller RC (2006) Functional bowel disorders. Gastroenterology 130: 1480-1491

9. van der Veek PPJ, Schots EDCM, Masclee AAM (2004) Effect of neurotensin on colorectal motor and sensory function in humans. Dis Colon Rectum 47:210-218

10. Kamerling IM, Burggraaf J, van Haarst AD, Oppenhuizen MF, Schoemaker RC, Biemond I, Jones R, Heinzerling H, Cohen AF, Masclee AAM (2003) The effect of motilin on the rectum in healthy volunteers. Br J Clin Pharmacol 55(5):538-543

11. van der Veek PPJ, Steenvoorden M, Steens J, van der Schaar PJ, Brussee J, Masclee AAM (2007) Recto-colonic reflex is impaired in patients with irritable bowel syndrome. Neurogastroenterol Motil 19:653-9

12. Delgado-Aros S, Camilleri M, Garcia MA, Burton D, Busciglio I (2008) High body mass alters colonic sensori-motor function and transit in humans. Am J Physiol Gastrointest Liver Physiol 295:G382-8 